\title{
Research on the Interactive Training Pattern and the Applications on the Modern Emergency Rescue Training Activities
}

\author{
Zhang Hua \\ School of Nursing, Central South University, \\ Chang Sha, China, 410013 \\ Hainan Medical University, Haikou, China, 571199 \\ email: zhanghuashelley@ hotmail.com \\ He Guoping * \\ School of Nursing, Central South University, \\ Chang Sha, China, 410013 \\ email: hgpcsu@aliyun.com \\ Xu Baichao \\ Hainan Medical University, \\ Haikou, China, 571199 \\ Zhang Na \\ Hainan Medical University, \\ Haikou, China, 571199 \\ Lv Chuanzhu \\ Hainan Medical University, \\ Haikou, China, 571199
}

\begin{abstract}
In this paper, we conduct research on the interactive training pattern and the applications on the modern emergency rescue training activities. In emergency rescue training linkage the ecological communities, important is not gathered themselves together, and not the size of the momentum and the strength that is not the amount of emergency rescue training linkage performance, important is the ecological communities each organic elements in the function of the cohesion, lies in the relationship between the organic elements in ecological communities, the construction and the development is the specific ecological communities in action elements of emergency rescue training, joint quality lies in the emergency rescue training. These contents are involved in the emergency rescue training linkage management model of concrete. Under this basis, we integrate the condition of the Hainan Medical School to propose the corresponding suggestions that will enhance the performance.
\end{abstract}

Keywords- Interactive Training, Applications, Pattern, Emergency Rescue, Training Activities.

\section{Introduction}

With the quickening of the urban development and construction, urban floating population increase, the population increased liquidity, all kinds of traffic accidents, construction damage and accident harm accident are increasing, frequent injuries to see a doctor. To hurt the wounded rescue process, because of its sudden strong as the wounded quantity, injury complicated, seriously, therefore, within the hospital on the emergency rescue system put forward the higher requirements. Public emergency occur and therefore, how to safely admit to hurt the wounded, orderly, efficient has always been an important task to all levels of hospital. Nursing is an important part of hospital emergency treatment work, must establish perfect and scientific system of emergency treatment that can improve the ability of nurses of the emergency rescue which is important [1-3].

According to the literature review, the settlement and the architecture of the emergency steps can be organized as the follows. (1) The mobile group, this group by the emergency department head nurse group leader, nurses by the emergency center, distribution of motor according to need to the first aid group, to cooperate to complete the critical catheter, endotracheal intubation, and using the breathing machine work, complete professional operation, for ensure that patients receive treatment in time 
and accurately. (2) Critically ill patients group, the group leader by the head nurse's role as a leader in the ICU, cooperate a doctor to complete the critical catheter, endotracheal intubation, guardianship, and using breathing machine work, complete professional operation, to ensure timely and accurate in the critical patients get treatment. (3) Logistics group that is composed of a distribution center hospital personnel, flexible dispersed to the each group, to follow the arrangement of the group leader, assist to escort the injured on inspection, emergency surgery, hospitalization, and the inspection report, etc.

Base on the survey, we summarize the state-of-the-art training patterns as the follows. (1) The case import, in the interpretation of each content, to undertake import with real case, this paper from two aspects of positive and negative learning that the necessity of the common rescue skills to stimulate students' enthusiasm for learning. (2) Using a variety of the teaching methods multimedia courseware and teaching operation all have their respective advantages, combination according to the actual situation in the teaching. For example, in the interpretation of cardiopulmonary resuscitation (CPR), the teacher and the application of multimedia courseware, teaching on the side in cardiopulmonary resuscitation (CPR) simulation, interspersed with operating video, leave some time by students back and operating in practice. (3) Role play scenario simulation on airway foreign body after obstruction, scenario simulation training. One of student plays a patient the teacher plays the rescue, the airway obstruction call for help with technique of scene that is advantageous to the student aid technology. Therefore, in this paper, we conduct research on the interactive training pattern and the applications on the modern emergency rescue training activities that will be discussed in detail in the later parts.

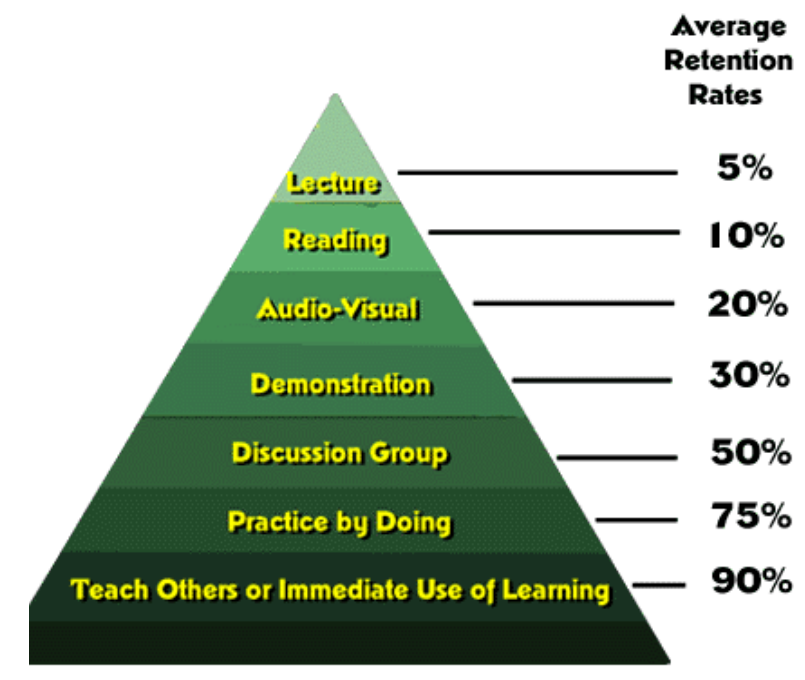

Figure 1. The Properties of the Interactive Training Pattern

\section{The Proposed Methodology}

The Features of the Modern Emergency. Hospital has a key role in the dealing with the public emergency, for to hurt the injured trauma care in the hospital is the key links of the treatment system. At present, the hospital has not been formed within the trauma of the unified model, according to their different hospital characteristics in the different forms of trauma, the treatment effect have significant difference, but for a batch of the wounded rescue are more confined to the working experience and understanding. How to promote to hurt the wounded treatment work of the standardized scientific and standardized and the rational allocation of the care resources, optimizing the rescue process is still an important subject which 
needs further modification and optimization. Therefore, the training of the emergency rescue will optimize the procedures with the listed aspects.

- Improving the first-aid nursing ability. Build system of emergency rescue system is effectively cope with the various emergencies the key to improve the general ability of emergency rescue. Establishing perfect emergency management organization can be timely to make the work of emergency organization, coordination and control, ensure the smooth progress of the rescue.

- Improve the quality of the care of critically ill patients. Using the experience of the emergency nursing, the establishment of severe trauma patients rescue the green channel, application of information systems for identification of critically patients, general condition evaluation and quality control, improve emergency ward, operating room between the patient's safe transport, transfer, etc., not only optimize the nursing process, also promoted the critically ill patient care quality further improved [4-5].

- To promote the construction of nursing team with the establishment of emergency response teams, and provides the skills outstanding nurses talents platform, promote the stability of the nursing cadre and nursing talented person's echelon construction, which laid a foundation for the further improve the level of nursing security.
The Rescue Work in the Other Countries. At present, many countries in Europe and the United States has established a perfect emergency system, and through the laws and regulations for public emergency rescue training institutions, training time, training content and so on made clear rules and requirements. Australia thinks that community volunteer first aid knowledge education and training is the key to improve the ability of first aid and the volunteers to 10 hours of training that is one of the training task of ambulance service center, trained in Australia public accounted for 50\%. The British government with a special focus on the citizen, the police first aid knowledge education, makes the master first aid skills, first aid knowledge penetration rate of $33 \%$, and all persons involved in the rescue work must receive professional training from the 12 weeks. In Sweden, the bearing of the Red Cross is a public first-aid training institutions, its 58000 people a year to attend first aid training and the national emergency training can be unified teaching material and the emergency plan manual, all rescuers must pass a standardized training to the qualified for the rescue work.

The United States are of the utmost importance to popularize first aid knowledge education, in 1966 the American heart association began to advocate general popularization of cardiopulmonary resuscitation in public primary resuscitative methods, before brought to the house rules that the first aid training issue police, drivers, firefighters, the teachers and schools must receive cardiopulmonary resuscitation and the field rescue to save the training. 


\section{INCIDENT COMMAND SYSTEM}

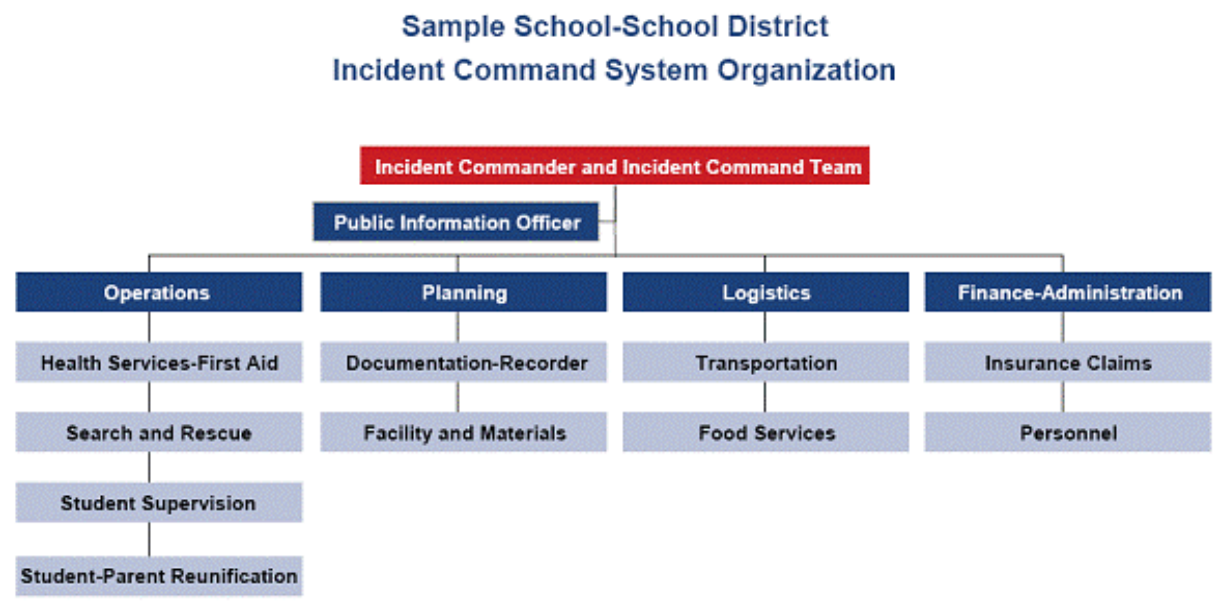

Figure 2. The Modern Emergency Rescue Training Activities

The Interactive Training. Emergency rescue training linkage management refers to the whole society and emergency rescue training work has a direct or indirect correlation department, unit, and the individual industry, through the good communication and effective exchange of information, the contact interaction, resources integration and collaboration to promote the participation, training and organization of regular operation mechanism. In a sense can call emergency rescue training linkage management linkage mechanism for the training of emergency rescue [6].

For management mode and mechanism, from the point of its real performance, both need to pass a certain system, specification, or policy such as dominant related content, become a part of the social appearance but in terms of its internal both are hidden behind the operation logic and rules the explicit representation belongs to the level of abstraction that can be organized as follows.

- College nursing students training. Disaster rescue training of general nursing students of the undergraduate course and specialized subject, to simulate the scene practice supplemented, theory of classroom teaching which make the nursing students to master relevant theoretical knowledge and disaster rescue different types of rescue skills, such as the cardiopulmonary resuscitation (CPR), the on-site emergency technology and post-disaster psychological crisis intervention, and other commonly used basic technology.

- The backbone teacher training. Ambulance in imparting knowledge and skills at the same time, increase the effective teaching method and content of practice teaching such as teaching management, cultivate their teaching ability and management ability, making it the ability of disaster rescue trainers and expand the knowledge and the skills of disaster rescue and disaster nursing teaching level unceasing enhancement, cultivating high-quality talents in the field of the general and the traditional disaster nursing.

- Senior professional personnel training. In the framework of the first-aid training outline, as the professional, teaching system that the training should focus on disaster nursing management, 
psychological crisis intervention and statistical basis, etc.

\section{The Hainan Medical Emergency Rescue}

Training. Under the leadership of the leading group for emergency rescue training, the school organization of personnel, the educational administration department, studies ministry, the youth corps committee and so on various secondary, the teaching administrative department actively involved in the specific work, school students, and each student community as a volunteer to assist training. Primary emergency rescue knowledge training, in order to improve the penetration and rescue training and certification rate become the highest standard of the domestic colleges, results obtained, for the following reasons.

- Preliminary build a long-term mechanism. Number of the emergency rescue training work is broad and training duration is long that represent the very difficult task.

- School leaders direct participation, the comprehensive plan for emergency rescue training specifications and the thorough development as well as the training system and full funding safeguard smoothly [7].

- Opened up a column, the school website, radio and other media publicity that save the rescue emergency rescue knowledge on the disaster prevention and mitigation, and promptly take emergency rescue training in our school by the progress of the work of the Hainan TV station, People's Daily, the south China sea, Hainan medical college campus network television, the Internet means such as the propaganda to improve the initiative and self-consciousness of the teachers and students to participate in the learning and training in our school.

To pay equal attention to scientific research and practice, constantly seeking innovation in practice that many ways to popularize knowledge of emergency rescue especially need to point out that the emergency rescue training in our comprehensive and the thorough development at the same time, the school actively to provide training services to the public. Innovative measures, on the one hand, it can consolidate students' emergency rescue knowledge, skills, hands-on ability increased significantly; On the other hand also to fully excavate students excellent and advanced molecular join family to the emergency rescue training and training promotion effect. Our school Red Cross volunteers overcome site, weather and other difficulties, sacrifice personal time to rest, weekend or evening, guarantee the emergency rescue training for all the work smoothly [8].

In the future, our further work will be focused on the listed aspects. (1) Assist the province Red Cross guidance at all levels of the Red Cross in emergency rescue knowledge popularization and the general basic training. (2) Emergency rescue training standardization construction. (3) Make training syllabus and ambulance training materials based on the literature reviews.

\section{Conclusion}

In this paper, we conduct research on the interactive training pattern and the applications on modern emergency rescue training activities. Emergency rescue training linkage management focuses on the linkage management is actually on the basis of technical boundary and social boundary will integrate all the elements of this open ecosystem harmonious, interactive mode and process, which contains both the horizontal consolidation and also contains the vertical extension, will a variety of resources integration and sharing, in order to improve the linkage management efficiency. Elements from the emergency rescue training linkage, a detailed analysis of technology and social boundaries, the body of the emergency rescue training linkage not only include the administrative organs and organizations in different areas, including the related market power and social power. Under these guidelines, we later propose our unique 
perspectives on the issues that will be meaningful and innovative.

\section{Acknowledgement}

National Natural Science Foundation of China （Grant No.71263014）; The Project Supported by the School Committee of the Communist Youth League in China ( Grant No. 2015YB084) ; Philosophy and Social Science Planning Project of Hainan Province (Grant No. HNSK（QN）15-88）; Education Foundation of Hainan Province (Grant No.Hnky2015-38); Funds for the Medical Humanities Research Base of Hainan Province （Grant No.QRYPT201507）

\section{References}

[1] Dorman, Terri, et al. "Carers' training in the administration of emergency rescue medication: Terri Dorman and colleagues outline results from a survey of how medications to manage seizures in people with learning disabilities and epilepsy are administered." Learning Disability Practice 18.4 (2015): 27-30.

[2] Dwyer, Kristin, et al. "Opioid education and nasal naloxone rescue kits in the emergency department." Western Journal of Emergency Medicine 16.3 (2015): 381.

[3] Lei, B. W., et al. "The design of rescue drills for coal mine explosion accident and the implementation research of virtual reality." Computer, Intelligent Computing and Education Technology (2014): 209.

[4] Nagatani, Keiji, et al. "Emergency response to the nuclear accident at the Fukushima Daiichi Nuclear Power Plants using mobile rescue robots." Journal of Field Robotics 30.1 (2013): 44-63.

[5] Jones, Jerrilyn, et al. "emergency medical services response to active shooter incidents: Provider comfort level and attitudes before and after participation in a focused response training program." Prehospital and disaster medicine 29.04 (2014): 350-357.

[6] Higuchi, Hiroko, et al. "Disaster Detection by Statistics and SVM for Emergency Rescue Evacuation Support System." Parallel Processing Workshops (ICCPW), 2014 43rd International Conference on. IEEE, 2014.

[7] Wiesner, Wojciech, and Bogusław Kowalewski. "The Analysis the Emergency Protection of Water Reservoirs in Olsztyn." Polish Hyperbaric Research 52.3 (2015): 59-66.

[8] Amanatiadis, Angelos, et al. "The avert project: Autonomous vehicle emergency recovery tool." Safety, Security, and Rescue Robotics (SSRR), 2013 IEEE International Symposium on. IEEE, 2013. 